Dermatology 2008;216:279

DOI: $10.1159 / 000113947$

\section{Multiple Lentigines Confined to Resolving Psoriatic Plaques in a Patient Treated with Adalimumab}

\author{
Jorge Santos-Juanes ${ }^{\mathrm{a}}$, Pablo Coto a , Susana Mallo ${ }^{\mathrm{a}}$, \\ Cristina Galachec, José Sánchez del Río ${ }^{\mathrm{a}}$, J.C. Torre ${ }^{\mathrm{b}}$ \\ a Servicio de Dermatología II, Hospital Universitario Central de \\ Asturias, y ${ }^{b}$ Servicio de Reumatología, Hospital Monte Naranco, \\ Facultad de Medicina, Universidad de Oviedo, Oviedo; \\ 'Servicio de Dermatología, Hospital de Cabueñes, Gijón, Spain
}

\author{
Key Words \\ Lentigines, multiple $\cdot$ Psoriatic plaque $\cdot$ Adalimumab
}

Hyperpigmented lesions confined to resolved psoriatic plaques have been rarely reported and have been previously described as lentigines, naevus spilus hyperpigmentation and speckled pigmentation [1-3]. We present the case of a 55-year-old woman with psoriasis who developed lentigines on resolving lesions while receiving adalimumab treatment.

\section{Report of a Case}

Our patient had suffered from chronic plaque psoriasis on the elbows and knees for more than 25 years and had been successfully treated with various topical treatments including steroids, tacalcitol and calcipotriol. She had been free of lesions while under treatment. In the 2 previous years she had also received methotrexate for psoriatic arthritis but with poor control of the disease. In August 2006, she started adalimumab $40 \mathrm{mg}$ subcutaneously once every 15 days. At this time she presented psoriatic plaques on the elbows and knees. After 2 months of treatment, she was admitted to our service as the psoriatic plaques had disappeared and new lesions had appeared. On physical examination, she had light and brown regular lentigines over the previous sites of the psoriatic plaques (fig. 1), which were confirmed as such on histological examination. Treatment was continued, and no more lesions or changes in the morphology or colour of the lentigines were seen. Phototherapy was not performed in this patient.

\section{Comment}

Multiple large solar lentigines on the upper back have been described as clinical markers of past severe sunburn [4]. The majority of the lentigines in resolving psoriatic plaques are described following PUVA or UVB. In the few previous cases reported the underlying mechanism for this kind of lesion is unknown. This pigmentation can arise without phototherapy, and it has been suggested to be an unusual type of postinflammatory hyperpigmentation [5]. In our case the chronological association between the use of adalimumab and the development of the lesions may suggest that adalimumab is of aetiological relevance to their development and presumably has some effect on melanocyte biology. We do not know if the formation of lentigines could be: (a)
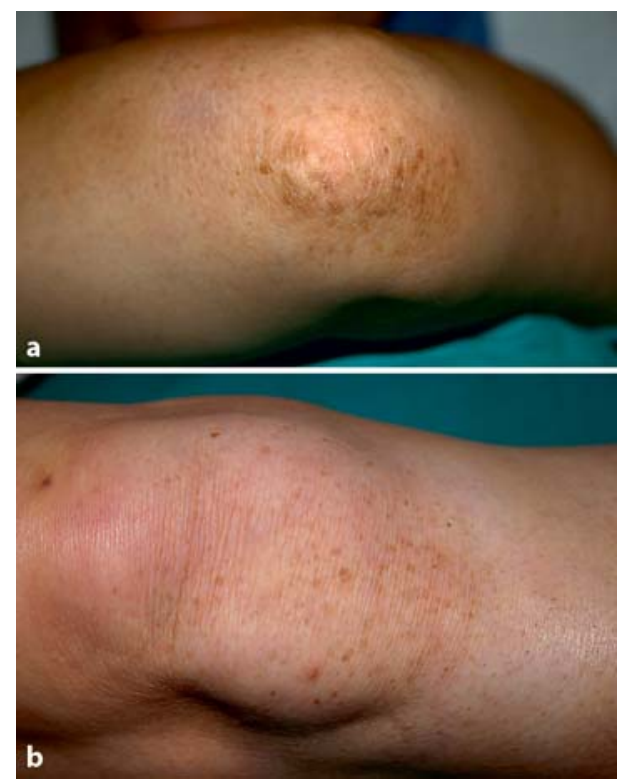

Fig. 1. Lentigines can be seen on the elbows (a) and knees (b) where the previous psoriatic plaques existed.

drug induced, (b) the result of the immunosuppressive action of adalimumab, because systemic immunosuppressants are reported to cause an increase in melanocyte activity, or (c) might simply be a postinflammatory hyperpigmentation.

On the other hand, melanocytes may be involved in the psoriatic process in several ways: there have been several reports suggesting an association between chronic plaque psoriasis and vitiligo, and the absence of lentigines in psoriatic plaques has been presumed to be the result of selectively destroyed melanocytes by the psoriatic process [6].

The clinical implication of our finding is uncertain. Overall, careful surveillance will be required in those patients receiving biological treatments.

\section{References}

1 Helland S, Bang G: Nevus spilus-like hyperpigmentation in psoriatic lesions during PUVA therapy. Acta Derm Venereol (Stockh) 1980;60: 81-83.

2 Poskitt BL, Wilkinson JD, Wojnarowska FT, Chia YC: Patchy speckled bath PUVA pigmentation. J R Soc Med 1993;86:665-666.

3 Mitra A, Yeung R, Sheehan-Dare R, Wilson CL: Lentiginous hyperpigmentation confined to resolved psoriatic plaques and treated with a Q-switched ruby laser. Clin Exp Dermatol 2005;31:281-305.

4 Multiple large solar lentigos on the upper back as clinical markers of past severe sunburn: a case control study. Dermatology 2007;214:25-31.

5 Rogers M: Multiple lentigines confined to a resolving psoriatic plaque treated without phototherapy. Clin Exp Dermatol 1994;19:380-382.

6 Mendonca CO, Holmes S, Burden AD: Absent lentigines in psoriatic plaques. Br J Dermatol 2002;147:1020-1046.

\section{KARGER}

() 2008 S. Karger AG, Basel

Fax +41 613061234

E-Mail karger@karger.ch

www.karger.com
Accessible online at:

www.karger.com/drm
Dr. Jorge Santos-Juanes Servicio de Dermatología II

Hospital Universitario Central de Asturias, C/Julian Clavería s/n ES-33006 Oviedo (Spain)

Tel. +34 626055 102, Fax +34 985208 497, E-Mail jsantosj@hca.es 